\title{
ESTUDIO EXPLORATORIO DE LA VARIABILIDAD RADIAL Y APICAL DEL TAMAÑO Y FRECUENCIA DE LOS CANALES RESINÍFEROS EN PINO RADIATA
}

\author{
PRELIMINARY STUDY OF THE RESIN CANALS IN RADIATA PINE
}

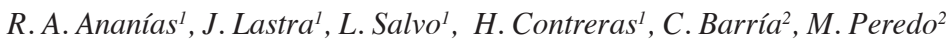

\section{RESUMEN}

En este trabajo se cuantifica la variabilidad radial y apical de los canales resiníferos de pino radiata (Pinus radiata) de 14 años.

Se determinó la frecuencia y el tamaño de los canales resiníferos en muestras microtomadas usando un software comercial. Las muestras fueron extraídas de árboles en pie presentando tres niveles diferentes de resinación externa, es decir, moderada/leve, e intensa. Se evaluó la variabilidad de los canales resiníferos según la posición radial a tres diferentes alturas: base, DAP, y al comienzo de la copa del árbol.

Los resultados muestran que la variación radial y apical del tamaño y la frecuencia de canales resiníferos aparece débilmente correlacionada con el nivel de intensidad de resinación observada en árboles en pie de pino radiata.

Palabrasclave:Frecuenciay tamañode canales resiníferos, Áreadecanales resiníferos,Resinación,Coníferas.

\begin{abstract}
Radial and apical variation in resin canals of radiata pine were studied. The frequency and size of resin canals were examined, using microscopic techniques from a commercial software. Wood samples from standing trees were taken, showing three different levels of external resin-bleeding intensity : moderate, light and intense. The variability of the resin canals were examined according to the radial position at three different heights ; bottom, breast height diameter and at the beginning of the tree top. The results show that the apical and radial variation of the size and frequency of resin canals appeared weakly correlated with the intensity level of the tree resin-bleeding of the standing radiata pine trees.
\end{abstract}

Keywords: size and frequency of resin canals, Resin canal area, Resin-bleeding, Softwoods.

\section{INTRODUCCIÓN}

El manejo intensivo de las plantaciones esta orientado a la obtención de productos forestales con un mayor valor agregado; sin embargo, la aparición de defectos en los productos finales, puede ocasionar pérdidas económicas importantes por desclasificación o reprocesamiento de los mismos.

Entre los defectos destacan, los originados durante la etapa de crecimiento de los árboles (venas y bolsas de resina, pecas y grietas internas). Estos defectos están dentro de la madera y sólo

\footnotetext{
${ }^{1}$ Universidad del Bío-Bío, Facultad de Ingeniería, Departamento de Ingeniería en Maderas. Concepción. Chile

${ }^{2}$ Bioforest. Concepción. Chile

Autor para correspondencia: ananias@ubiobio.cl

Recibido: 05. 04. 2010 Aceptado: 11. 06. 2010.
} 
son observados cuando la madera es transformada. Según Harding et al. (2006), las consecuencias económicas por la presencia de defectos resinosos en madera aserrada proveniente de plantaciones de Pinus elliotti, varia considerablemente y afectan alrededor de un $2 \%$ de la madera aserrada verde proveniente de buenos sitios, y puede llegar al $40 \%$ cuando la materia prima proviene de sitios no óptimos.

En pino radiata, según Gazo et al. (2000a-b) la presencia de bolsas de resina se ve favorecida por la poda. Beauregard et al. 1999, encuentran un alto grado de heredabilidad de las pecas, pero una baja heredabilidad en las bolsas de resina y corteza. Kumar (2004) no evidencia correlación alguna entre la densidad de la madera y el sangrado de resina en pino radiata. Por otra parte, Keey et al. 2000 indican que el sangrado de resina es favorecido durante el secado de pino radiata a una temperatura superior a $\operatorname{los} 80^{\circ} \mathrm{C}$.

Las bolsas de resina son separaciones de las traqueidas, cuyas causas de formación no son bien conocidas (Kininmonth and Whitehouse 1991). No obstante Cown (1973) plantea que estos son formados en el cambium como consecuencia de stress hídrico y/o cavitación de la resina en los canales resiníferos. Esto es comúnmente asociado a la presencia de anillos falsos, formándose en bandas tangenciales en la dirección de menor resistencia como en la zona de canales resiníferos. De acuerdo con Clifton (1969) la variabilidad radial y apical de las bolsas de resina en pino radiata es heterogénea, el número de bolsas de resina aumenta con la altura del árbol hasta alrededor de los $8 \mathrm{~m}$. y luego tiende a mantenerse constante, y desde la médula a la corteza aumenta inicialmente y luego tiende a disminuir. En Picea abies, en cambio, las bolsas de resina se distribuyen aleatoriamente en el interior de los árboles, pero son más grandes y abundantes hacia la copa del árbol y desde médula a corteza (Gjerdrum and Bernabei 2007). Ridoutt et al. (1999), muestran que dependiendo de la procedencia de lo árboles se puede observar una interrelación moderada entre la resinación (sangrado de resina) en el extremo de las trozas y los bolsillos de resina en la madera.

El pino radiata es una especie que se caracteriza por la presencia de canales resiníferos, y estos son formados postcambialmente por la separación de las células epiteliales (Panshin and de-Zeeuw 1980). Los canales resiníferos disminuyen en frecuencia y tamaño desde la médula a corteza (Koch 1973). Por lo contrario, según Yang et al. (2007), el tamaño de los canales resiníferos aumenta desde la médula a la corteza, pero disminuyen su frecuencia. La presencia de canales resiníferos aumenta moderadamente la resinación de la madera y esta débilmente correlacionada con los bolsas de resina y la resinación externa en el árbol. Por otra parte, la presencia de canales resiníferos no parece estar asociada a la presencia de grietas internas entre los anillos de la madera de pino radiata (Nair et al. 2009). Se ha observado niveles variables de intercomunicación entre canales resiníferos longitudinales o transversales (Lapasha y Wheeler 1990). Esta interrelación puede favorecer el transporte de resinas entre estos tejidos longitudinales y transversales, en este sentido el sangrado de resina (resinación) puede ser favorecido tal como es reportado por Singh et al. 2007.

En este trabajo se estudia la variación radial y apical de los canales resiníferos y su relación con la intensidad de resinación observada en árboles en pie de pino radiata.

\section{METODOLOGÍA}

Se estudiaron 6 árboles de pino radiata (Pinus radiata) de 14 años de edad, creciendo en la zona de Traiguén, en el sector Reñico. Los árboles fueron seleccionados en pie (con igual banda diamétrica y espaciamiento de 600 y 1600 árboles/ha. Los niveles de intensidad de resinación (observada por un clasificador-experto de la empresa industrial al que pertenece el rodal) estudiados fueron: sin resina (árboles testigos), resinación moderada/leve e intensa (Tabla 1). 
Tabla 1: Detalles de los árboles seleccionados para este estudio

\begin{tabular}{|c|c|c|c|}
\hline Árbol & Espaciamiento & Resinación & DAP (cm) \\
\hline 1 & 1600 & Sin resinación (testigo) & 23.2 \\
\hline 2 & 1600 & Leve & 23.1 \\
\hline 3 & 1600 & Intensa & 24.5 \\
\hline 4 & 600 & Sin resinación (testigo) & 29.8 \\
\hline 5 & 600 & Moderada & 26.9 \\
\hline 6 & 600 & Intensa & 31.5 \\
\hline
\end{tabular}

De cada árbol se obtuvieron dos rodelas (discos) a tres alturas, h0: en la base, h1: DAP, h2: HCCV (altura comienza copa viva). Para la determinación de las características anatómicas microscópicas se prepararon cortes microtómicos de acuerdo a Chaffey (2002). Luego los cortes fueron analizados radialmente (desde médula a corteza) anillo por anillo, a través de análisis de imágenes utilizando el programa WinCELL versión 5.1. Las mediciones de los canales resiníferos fueron realizadas en la dirección radial considerando la nomenclatura de canal resinífero descrita por Wiedenhoeft y Miller (2002). Se registró el diámetro radial y numero de canales resiníferos, luego se calcularon el área (tamaño de los canales resiníferos) y el número de canales $/ \mathrm{mm}^{2}$ (frecuencia). Se realizaron 50 mediciones de la frecuencia de los canales resiníferos en cada posición.

Los factores variables fueron: 3 intensidades de resinación x n posiciones radiales (cada 2 cm) x 3 alturas del árbol (Base, DAP, HCCV). En la densidad de plantación de 600 árboles/ha, se recogieron 4 posiciones en la altura (Base, DAP, HCCV y 75\% de la altura total del árbol). Se realizó un análisis de varianza para comparar los resultados según posición radial y apical empleando un software comercial. Se realizó la prueba de Shapiro Wilks para probar normalidad de los valores medidos y la homogeneidad de la varianza fue confirmada con el test Bartlett y de Hartley. Se realizó una transformación a los datos, mediante la prueba Kruskal-Wallis, porque los mismos no mostraron normalidad y homogeneidad de varianza.

\section{RESULTADOS Y DISCUSIÓN}

\section{Variabilidad de la frecuencia de canales resiníferos}

En la Figura 1 se presenta la variabilidad radial (posiciones 1 a 9$)$ y apical $\left(h_{0}, h_{1}, h_{2}, h_{3}\right) d e$ la frecuencia de los canales resiníferos.

En los árboles sin resinación (testigos), se observa una mayor frecuencia de los canales resiníferos en una densidad de plantación de 600 árboles/ha, esto es, la variación radial es aproximadamente desde 1.5 a 1 canales resiníferos $/ \mathrm{mm}^{2}$ (Figura 1d); a una densidad de plantación de 1600 árboles/ha, la variación radial es alrededor de 1 y 0.5 canales $/ \mathrm{mm}^{2}$ (Figura 1a).

En los árboles con nivel de resinación moderado/leve, se observa que la frecuencia de canales resiníferos es más baja en la densidad de plantación de 600 árboles/ha, esto es, de 0.8 a 0.5 canales/ $\mathrm{mm}^{2}$ (Figura 1e); a una densidad de plantación de 1600 árboles/ha, la variación radial de la frecuencia de canales resiníferos disminuye de 1 a 0.5 canales $/ \mathrm{mm}^{2}$ (Figura 1b). Esto pese a una mayor intensidad de resinación observada a una densidad de plantación de 600 árboles/ha. 
En los árboles con nivel de resinación intensa, la frecuencia de canales resiníferos es más alta para una densidad de plantación de 600 árboles/ha, es decir, la frecuencia decrece de 1.5 a 0.5 canales/ $\mathrm{mm}^{2}$ (Figura 1c); a una densidad de plantación de 1600 árboles/ha, la variación radial de la frecuencia de canales resiníferos disminuye de 1 a 0.5 canales $/ \mathrm{mm}^{2}$ (Figura 1e).

Se observa además, que la frecuencia de canales resiníferos disminuye con la altura del árbol, sin embargo, la misma no resultó significativa estadísticamente. A una densidad de plantación de 600 árboles/ha este comportamiento es aún más notable, que para una densidad de plantación de 1600 árboles/ha.

Las diferencias en la frecuencia de los canales resiníferos no fueron significativas estadísticamente en todos los casos analizados.
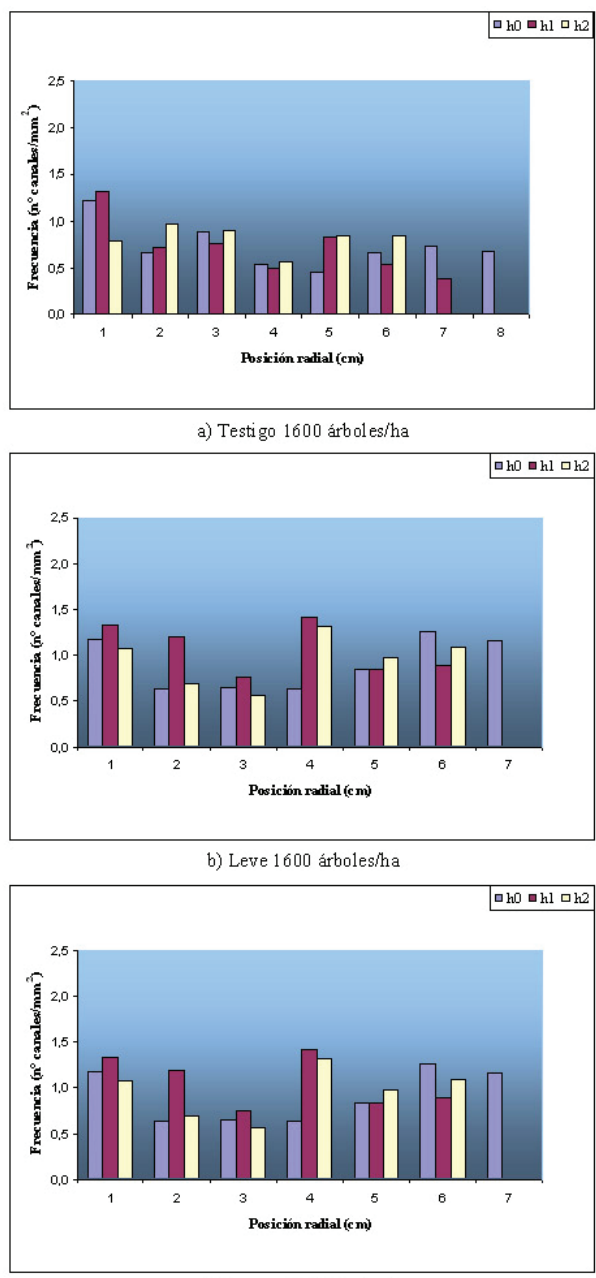

c) Intenso 1600 árboles/ha

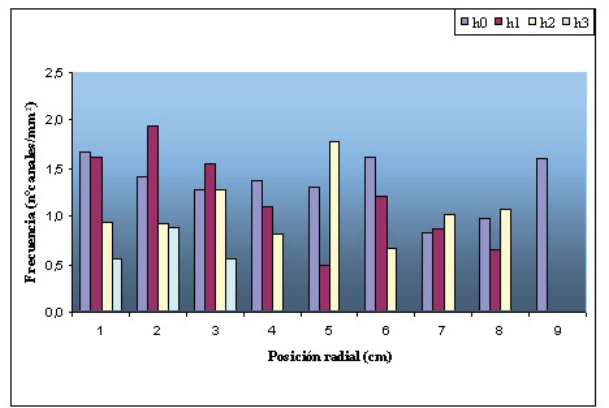

d) Testigo 600 árboles/ha

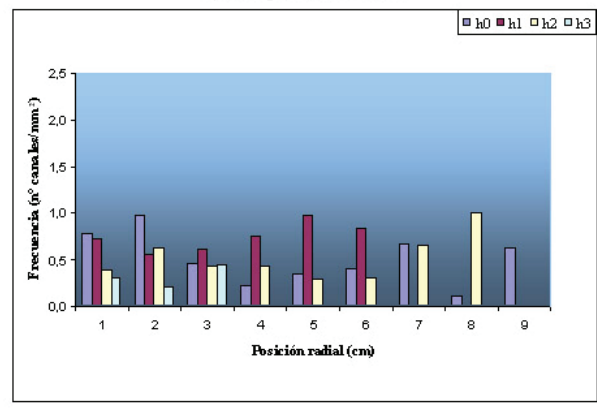

e) Moderado 600 árboles ha

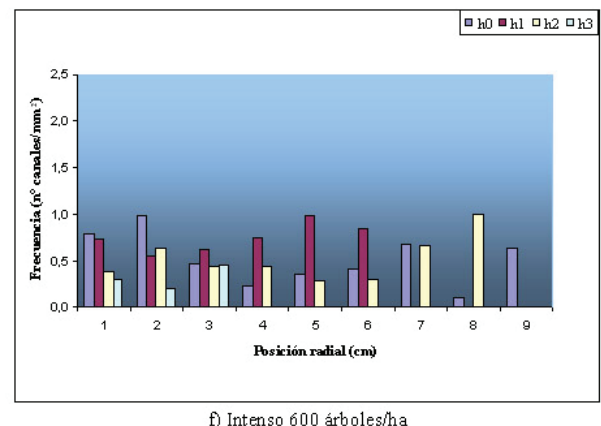

f) Intenso 600 árboles/ha

Figura 1: Variabilidad de la frecuencia de canales resiníferos en pino radiata (Posición radial: 1, 2, 3,..9. Posición en altura: $\mathrm{h}_{0}, \mathrm{~h}_{1}, \mathrm{~h}_{2}, \mathrm{~h}_{3}$ ).

\section{Variabilidad radial y apical del tamaño de los canales resiníferos}

En la Figura 2 se presenta la variabilidad radial (posiciones 1 a 9$)$ y apical $\left(h_{0}, h_{1}, h_{2}, h_{3}\right)$ del tamaño de los canales resiníferos. En general, se observa un aumento inicial del tamaño de canales resiníferos desde la médula a la corteza y un posterior descenso cerca de la corteza.

En los árboles sin resinación (testigos), se observa un mayor tamaño de los canales resiníferos en la densidad de plantación de 600 árboles/ha, es decir, la variación radial incrementa de $2 \times 10^{-2}$ a 3 
$\mathrm{x} 10^{-2} \mathrm{~mm}^{2}$ (Figura 2b); a una densidad de plantación de 1600 árboles/ha la variación radial aumenta de $1 \times 10^{-2}$ a $2 \times 10^{-2} \mathrm{~mm}^{2}$ (Figura 2a).

En los árboles con nivel de resinación moderado/leve, se observa que el tamaño de canales resiníferos es menor en la densidad de plantación de 600 árboles/ha, es decir, incrementa de $0.5 \times 10^{-2} \mathrm{a}$ $1.5 \times 10^{-2} \mathrm{~mm}^{2}$ (Figura 2e); a una densidad de plantación de 1600 árboles/ha la variación radial del tamaño de los canales resiníferos aumenta de $4 \times 10^{-2}$ a $5 \times 10^{-2} \mathrm{~mm}^{2}$ (Figura $2 \mathrm{~b}$ ). Esto pese a una mayor intensidad de resinación a una densidad de plantación de 1600 árboles/ha.

En los árboles con nivel de resinación intensa, el tamaño de los canales resiníferos es mayor para una densidad de plantación de 1600 árboles/ha, es decir, el tamaño de los canales resiníferos incrementa de $3 \times 10^{-2}$ a $4 \times 10^{-2} \mathrm{~mm}^{2}$ (Figura 2f); y a una densidad de plantación de 600 árboles/ha la variación radial del tamaño de los canales resiníferos aumenta de $1.5 \times 10^{-2}$ a $2.5 \times 10^{-2} \mathrm{~mm}^{2}$ (Figura $2 \mathrm{c}$ ).

Se observa además que el tamaño de los canales resiníferos disminuye con la altura del árbol. A una densidad de plantación de 600 árboles/ha este comportamiento es más notable, disminuyendo desde $2.2 \times 10^{-2}$ a $1.2 \times 10^{-2} \mathrm{~mm}^{2}$ desde la base a la copa. En cambio la variación apical para una densidad de plantación de 1600 árboles/ha disminuye de aproximadamente $2.5 \times 10^{-2} \mathrm{a} 1.6 \times 10^{-2} \mathrm{~mm}^{2}$. En todos los casos analizados, la diferencia en el tamaño de los canales resiníferos fue estadísticamente no significativa.

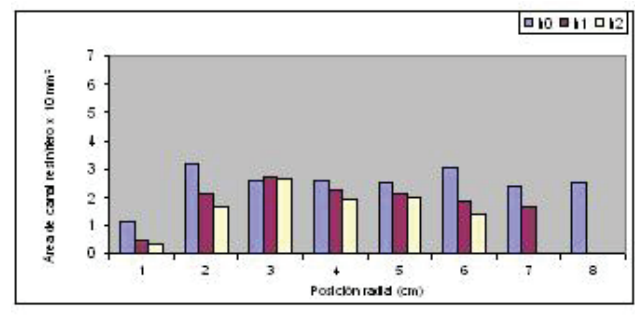

a) Testigo 1600 árboles/ha

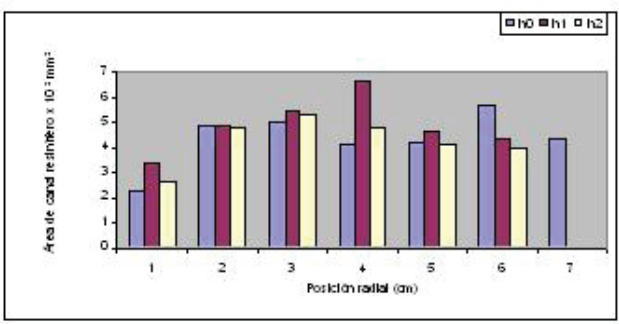

b) Leve 1600 árboles/ha

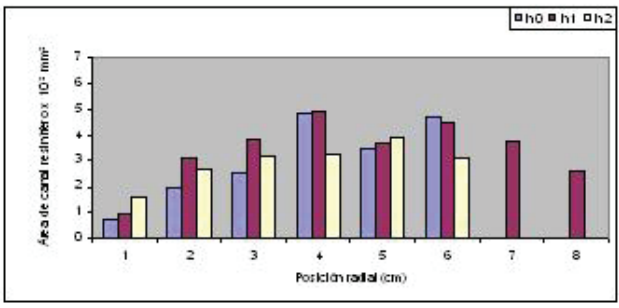

c) Intenso 1600 árboles/ha

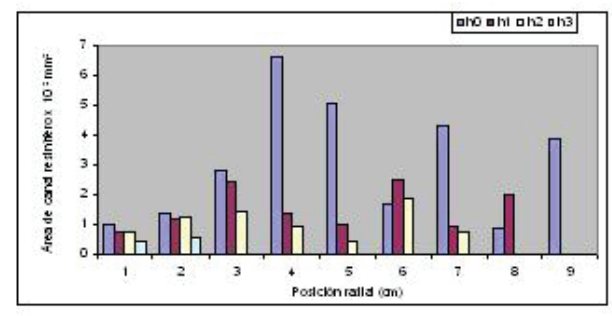

d) Testigo 600 árboles/ha

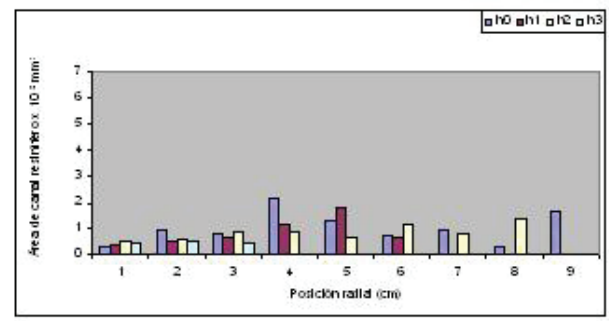

e) Moderado 600 árboles/ha

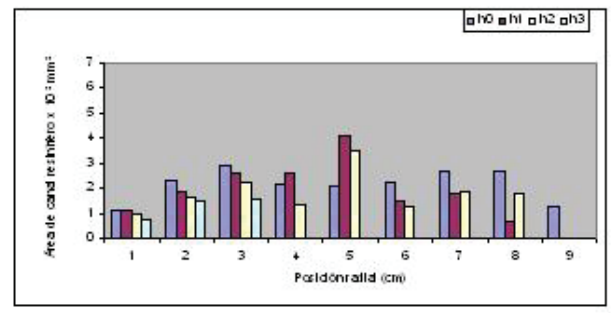

f) Intenso 600 árboles/ha

Figura 2: Variabilidad del tamaño de canales resiníferos en pino radiata (Posición radial: 1,2, $3, \ldots 9$. Posición en altura: $h_{0}, h_{1}, h_{2}, h_{3}$ ). 
Relación entre la variabilidad radial y apical de los canales resiníferos con la intensidad de resinación externa

Como observado anteriormente, se aprecia una variabilidad radial y apical del tamaño y la frecuencia de los canales resiníferos en pino radiata. Aunque esta variabilidad no es significante estadísticamente.

A una densidad de plantación de 1600 árboles/ha, la frecuencia de los canales resiníferos es máxima en los árboles con intensidad de resinación moderada a la altura del DAP y una posición radial intermedia (Figura 1b).

A una densidad de plantación de 600 árboles/ha, se observa una mayor frecuencia de canales resiníferos en todas las posiciones radiales y apicales del árbol testigo, pero notoriamente disminuyendo de la base a la copa.

Para la densidad de plantación de 1600 árboles/ha, un mayor tamaño de los canales resiníferos es observado en el árbol con intensidad de resinación leve, a la altura del DAP y en posición radial intermedia (Figura 2b y 3). Para una densidad de plantación de 600 árboles/ha, se observa un mayor tamaño de los canales resiníferos en la base del árbol de intensa resinación en la posición radial intermedia (Figura 2f y 4 ).

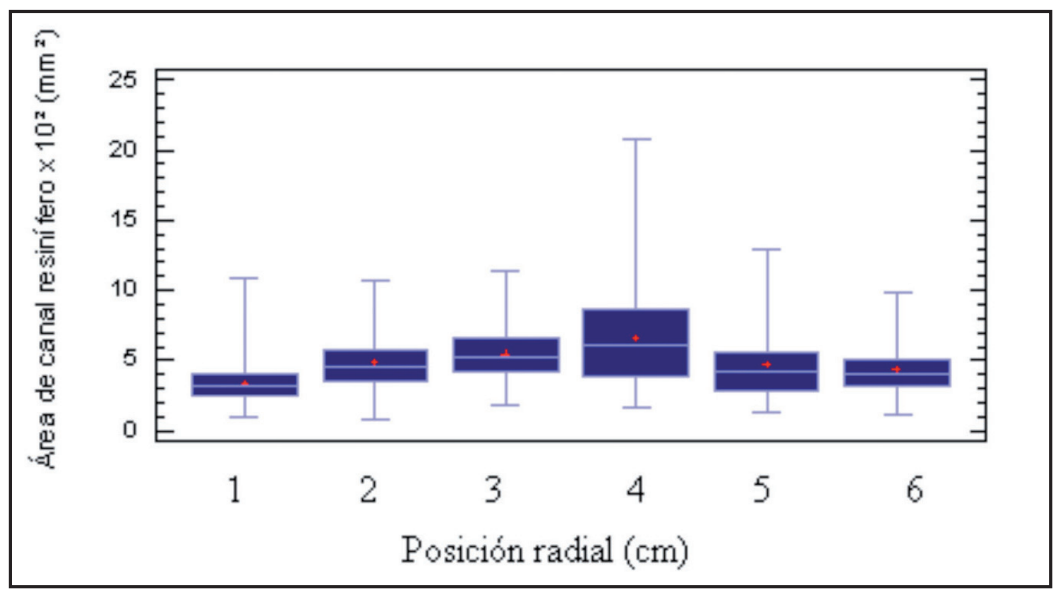

Figura 3: Variabilidad del tamaño de los canales resiníferos en el árbol resinación leve a la altura del DAP (1600 árboles/ha)

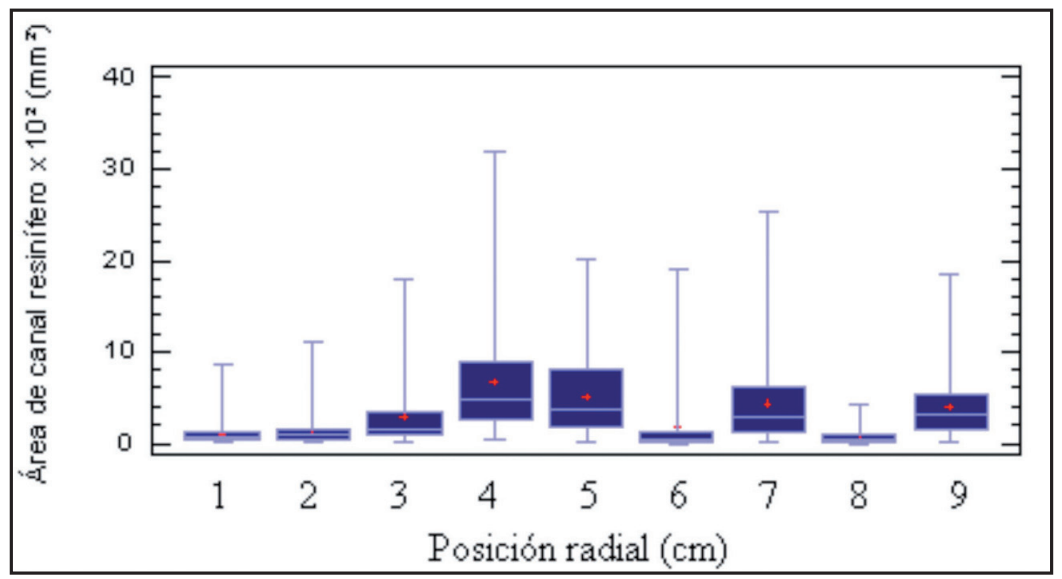

Figura 4: Variabilidad del tamaño de los canales resiníferos en el árbol resinación intensa a la altura del DAP (600 árboles/ha). 


\section{CONCLUSIONES}

La variación radial y apical del tamaño y la frecuencia de canales resiníferos aparece débilmente correlacionada con el nivel de intensidad de la resinación observada en árboles en pie de pino radiata. En una densidad de plantación de 1600 árboles/ha se observa mayor tamaño de canales resiníferos en árboles de resinación intensa. En una densidad de plantación de 600 árboles/ha se aprecia mayor frecuencia de canales resiníferos mientras mayor es la intensidad de la resinación de los árboles en pie.

La frecuencia de los canales resiníferos disminuye tanto en sentido radial y apical, esta variabilidad es más notable para árboles provenientes de una densidad de plantación de 600 (árboles/ ha).

El tamaño de los canales resiníferos aumenta en el sentido radial y experimenta un descenso cercano a la corteza, al contrario en el sentido apical los canales resiníferos disminuyen de tamaño en la medida que aumenta la altura, obteniéndose en general un mayor tamaño de canales resiníferos en las plantaciones de 1600 (árboles/ha).

La diferencia en la frecuencia de los canales resiníferos fue no significativa estadísticamente, lo mismo ocurrió con la diferencia en el tamaño de los canales resiníferos.

\section{BIBLIOGRAFÍA}

Beauregard, R.; Gazo, R.; Kimberley, M.; Turner, J.; Mitchell, S.; Shelbourne, A. 1999. Clonal variation in the quality of radiata random width boards. Wood and Fiber Science 31(3):222-234.

Chaffey, N. 2002. Wood microscopical techniques. In Wood formation in trees. Ch. 3. Ed. N. Chaffey. Taylor \& Francis, N. York. 17-40 p.

Clifton, N.C. 1969. Resin pockets in Canterbury radiata pine. N. Z. For. 14:34-49.

Cown, D. 1973. Resin pockets: Their occurrence and formation in New Zealand forests. N. Z. For. 18:233-251.

Gazo, R.; Beauregard, R.; Kimberley, M.; McConchie, D. 2000a. Incident of defects by tree charactertistics in radiata pine random-width boards. Forest Products Journal 50(6):83-89.

Gazo, R.; Beauregard, R.; Kimberley, M. 2000b. Influence of pruning and log height class on incidence of defects in radiata pine random-width boards. Forest Products Journal 50(9):28-31.

Gjerdrum, P.; Bernabei, M. 2007. Three-dimensional model for size and location of resin pockets of Norway spruce. Holz Roh Werkst. 65:201-208.

Harding, K.; Davis, J.; Copley, T.; Selleck, A.; Haslett, T. 2006. Resin defect impacts on the value of graded recovery and evaluation technologies for internal defect detection in slash pine logs. Report $\mathrm{N}^{\circ}$ PN04.3005. $41 \mathrm{pp}$.

Kininmonth, J.A.; Whitehouse, L.J. 1991. Properties and uses of New Zealand radiata pine. V. I. Wood properties. Forest Research Institute, Rotorua, New Zealand.

Keey, R.B.; Langrish, T.A.G.; Walker, J.C.F. 2000. Kiln-drying of lumber. Springer-Verlag, N. York. 
Koch, P. 1973. Utilization of the southern pines. Vol. I. The raw material. Agriculture Handbook $\mathrm{N}^{\circ}$ 420. USDA-Forest Service, USA.

Kumar, S. 2004. Genetic parameter estimates for wood stiffness, strength, internal checking and resin bleeding for radiata pine. Can. J. For. Res. 34:2601-2610.

Lapasha, C.; Wheeler, E. 1990. Resin canals in Pinus taeda, longitudinal canal lengths and interconnections between longitudinal and radial canals. IAWA Bull 11(3):227-238.

Nair,H.; Butterfield, B.; Jackson, S. 2009. Are rays and resin canals causal sites for intra-ring checking in the wood of Pinus radiata? IAWA 30(2):189-198.

Panshin, A.J.; de Zeeuw, C. 1980. Textbook of wood technology. Mc Graw Hill. N. York.

Riddout, B.; McConchie,D.; Ball, R. 1999. Predicting resin pockets in radiata pine logs from blemishes on log ends. Wood and Fiber Science 31(4):434-410.

Singh, A.; Dawson, B.; Turner, J. 2007. Resinous radiata. Understanding resin streaks and why does timber bleed resin. Wood Processing Newsletter 40,3pp.

Wiedenhoeft,A.; Miller, R.2002. Brief comments on the nomenclature of softwood axial resin canals and their associated cells. IAWA 23(3):299-303.

Yang, J.L.; Downes, G.; Chen, F.; Cown, D. 2007. Investigation of within-tree and between-site resin canal variation in radiata pine. Presented at the International Union of Forest Research Organizations (IUFRO) Conference, Division 5.01.01 - Biological control of wood quality. November 2007. Taipei, Taiwan. 